\title{
L'e-cigarette au miroir du risque tabagique
}

\section{Paul Vancassel et Alain Calmes}

\section{OpenEdition}

Journals

Édition électronique

URL : http://journals.openedition.org/communicationorganisation/4481

DOI : 10.4000/communicationorganisation.4481

ISSN : 1775-3546

\section{Éditeur}

Presses universitaires de Bordeaux

\section{Édition imprimée}

Date de publication : 1 juin 2014

Pagination : 49-58

ISBN : 978-2-86781-904-9

ISSN : $1168-5549$

\section{Référence électronique}

Paul Vancassel et Alain Calmes, «L'e-cigarette au miroir du risque tabagique », Communication et organisation [En ligne], 45 | 2014, mis en ligne le 01 juin 2017, consulté le 19 avril 2019. URL : http:// journals.openedition.org/communicationorganisation/4481; DOI : 10.4000/ communicationorganisation.4481 


\title{
L'e-cigarette au miroir du risque tabagique
}

\author{
Paul Voncossel ${ }^{1}$ et Alain Colmes ${ }^{2}$
}

La cigarette électronique a détrôné le tabac, pour nombre de fumeurs. Certains médecins la préconisent, même si son innocuité demeure à prouver. Les études scientifiques s'accordent cependant sur le fait qu'elle est nettement moins toxique que sa rivale. Les « vapoteurs » constituent une nouvelle tribu aux comportements paradoxaux et conservent, souvent, l'obsédant souvenir des rites tabagiques. Car, en un certain sens, vapoter, c'est encore fumer. Comment analyser ce phénomène en relation avec la théorie du risque ? Le risque tabagique constitue une des questions majeures de santé publique, avec l'addiction alcoolique et aux drogues. Pour aborder les questions posées par l'introduction de l'e-cigarette, peut-on se référer au modèle théorique d'Ulrich Beck, pour qui la science devient de plus en plus nécessaire mais de moins en moins suffisante pour définir la vérité ? Ou bien faut-il se référer à une approche originale de la logique de la répartition du risque qui appréhende l'individualisation ? Quelle éthique sous-tend l'usage de ce nouvel objet contemporain ? Certes, si l'on en croit la pastorale foucaldienne, le pouvoir étatique est dans son rôle, quand il prend en charge la santé du troupeau, un des éléments clefs de sa conductibilité. Il doit avoir l'œil sur tous et sur chacun (omnes et singulati), par l'entregent de ses micro-pouvoirs symboliques et effectifs. C'est aussi à cette figure de l'obéissance au pasteur que se réfèrent implicitement les nouvelles idéologies citoyennes teintées d'écologie. Et d'aucuns se rebellent, proclamant que le risque fait aussi partie de la vie. Le poète ne nous a-t-il pas avertis : « là où croît le danger crôit aussi ce qui sauve ». Injonction paradoxale à affronter, voire à commettre le pire, précisément pour son salut. Les nouvelles politiques hygiénistes empêcheraient, selon eux, le sujet d'assumer pleinement son destin. Alors, se demandent les réfractaires, appartient-il à une caste de censeurs de se positionner sur la diagonale du bien et du mal ?

1 Paul Vancassel est docteur en sciences de l'information et de la communication, chercheur associé au PREFics (Université Rennes 2) ; pvancassel@hotmail.com

2 Alain Calmes est professeur en sciences de l'information et de la communication, laboratoire PREFics (Université Rennes 2) ; alain.calmes.veyescaz@hotmail.fr 


\section{Dans l'ombre chaude de la cigarette}

L'herbe à Nicot a été vue très tôt comme un objet social et Molière vante ses bienfaits dans Don Juan. Certes, depuis sa découverte, le tabac a connu de multiples usages, mais la cigarette a généré l'engouement des foules, même si maintes études cliniques l'ont clairement dénoncée. Elle a sublimé les moments de détente de ses adeptes, mêlant son plaisir singulier aux zones érogènes supérieures ${ }^{3}$.

Elle a investi tout l'espace de l'agir communicant. Imprégnant les vêtements et les âmes, son odeur tenace se lovait, tel un parfum oriental, dans la moiteur des alcôves. Rares furent, au $\mathrm{XX}^{\mathrm{e}}$ siècle, les lieux la proscrivant. La cigarette devint la pierre angulaire de la pensée (Sartre en fit même une sorte de connecteur ontologique !) comme l'accomplissement de la pause. Un piège attractif, dont une des entrées était tapissée par les légendaires $\mathrm{P} 4$, cédées quelques centimes aux gamins, véritable pied dans la porte des gros paquets de 20. L'armée donnait des «Troupes » aux conscrits dès leur sélection lors des «trois jours». Ce potlatch a marqué le cinéma et la chanson. Et Bogart comme Gainsbourg en moururent... Le grand laxisme a duré tout un siècle, avant d'être disqualifié par la recherche du "risque zéro ». Soudain, la société sécuritaire stigmatise les fumeurs, ces suicidaires, armés d'une roulette russe, qui menacent la Sécurité Sociale. La question du tabagisme devient alors une véritable « construction sociale » dans l'imaginaire collectif, comparable à celles dont parle Claudine Burton-Jeangros ${ }^{4}$. Dans les années 1990, la mise en évidence du " tabagisme passif » a renforcé les argumentaires de santé publique, qui ont conduit à la loi Evin, puis à l'interdiction totale de fumer dans les lieux publics. La société civile, relayant l'orthopraxie étatique, a construit une représentation fortement négative du tabac, ce qui laisse un horizon illimité à la cigarette électronique.

\section{Le dispositif de la cigarette électronique}

C'est dans cet environnement hostile aux fumeurs que naît l'e-cigarette, nimbée d'un halo de pureté digne de la Naissance de Vénus de Botticelli. Ce dispositif électromagnétique ou électronique génère -on le sait - une vapeur destinée à être inhalée 5 . Celle-ci peut être aromatisée et contenir ou non de la

3 Voir Odile LESOURNE, La genèse des addictions, essai psychanalytique sur le tabac, l'alcool et les autres drogues, Paris, PUF, 2007.

4 Claudine BURTON-JEANGROS, «Des risques épidémiologiques aux politiques de santé ", in Face au risque, Genève, Georg, 2007, p. 190.

5 Le concept de la cigarette électronique a été élaboré par Herbert A. Gilbert en 1963, avec un brevet déposé en 1965, mais son produit ne fut jamais commercialisé. Il faut ensuite attendre 2003 pour voir apparaître le premier dispositif rendu public, destiné à simuler l'utilisation d'une véritable cigarette avec l'invention de Hon Lik, ancien pharmacien et ingénieur chinois, qui a déposé un brevet en 2005 pour une «cigarette sans fumée à pulvérisation électronique». Ce premier dispositif exploitait la technologie dite «ultra-sonique», et fut commercialisé en Chine en 2004 par la société Golden Dragon Holdings. Mais il est jugé complexe à industrialiser et coûteux. Finalement, le dispositif aujourd'hui le plus répandu a été inventé et breveté en 2009 par le chinois David Yunqiang Xin. Il s'agit de la technologie de vaporisation par résistance chauffante 
nicotine. À la différence de la fumée produite par une cigarette traditionnelle, cette vapeur n'a pas l'odeur du tabac et, selon les premières études scientifiques, contient des quantités de particules et substances cancérigènes ou toxiques beaucoup plus faibles. Son usage est néanmoins controversé

Un autre point de débat a été récemment soulevé, lors de la présentation d'un projet de loi au Parlement Européen (octobre 2013) : les lois anti-tabac doivent-elles - aussi - s'appliquer à la cigarette électronique ? Si certains commerces, notamment d'alimentation, étendent déjà l'interdiction de fumer à la cigarette électronique, des articles de presse signalent dans le même temps que de nombreuses entreprises autorisent leurs salariés à vapoter?

\section{Le charme discret de la cigarette électronique}

La cigarette électronique s'est diffusée dans la population d'abord par internet, puis par le relais de boutiques spécialisées, et sans aucune publicité dans les médias traditionnels. Son marketing s'appuie sur une approche nouvelle, que l'on pourrait qualifier d'« éco-citoyenne ", reposant sur la capacité des consommateurs à s'emparer de nouveaux produits et à en faire eux-mêmes la promotion. À travers quels usages et quelles représentations ce phénomène commercial tente-t-il de se développer ? Quel genre d'objet théorique constitue l'e-cigarette ? En fait, il s'agit d'un instrument plus complexe qu'il n'y paraît, entre phénomène de mode, dispositif technologique, substitut symbolique high-tech de la cigarette. Comment faut-il interpréter sa diffusion et son adoption si rapide par l'ensemble des couches sociales? Sont-elles dues à un phénomène de mode assimilable à l'attrait pour les « nouvelles technologies ", à un désir politique de s'opposer à l'industrie et aux multinationales du tabac, ou bien encore à une volonté de défier les interdits en continuant à fumer en public?

\section{Vers une approche individualisée du risque}

Revenons sur les différentes approches de la notion de risque. Pour aborder la question éthique, posée par l'introduction de l'e-cigarette, peut-on se référer à la notion de «risque» telle qu'elle est développée par Ulrich Beck dans $L a$ société du risque? Il semble que la réponse soit négative : cette approche de la « société du risque » est avant tout centrée sur l'étude des risques industriels ou environnementaux, tel que le risque nucléaire, ou les problématiques liées au génie génétique, à la production chimique ou encore aux émissions de

(«Electronic Nicotine Delivery System» ou ENDS). Source : wikipédia/cigarette électronique [en ligne].

6 La cigarette électronique a surtout fait l'objet de débats médiatiques dans la mesure où elle est commercialisée en France comme un «produit de consommation courante», qui échappe, de ce fait, au monopole des buralistes ou à celui des pharmaciens. Plusieurs organisations de santé (l'AFSSAPS en France en 2011 et l'OMS en 2013), ainsi que plusieurs rapports d'experts ont émis des recommandations contre son utilisation, notamment en raison de l'insuffisance de démonstration d'efficacité et de sécurité du produit, mais sans que la cigarette électronique soit jusqu'à présent soumise à davantage de réglementation en France.

7 Voir Le Monde.fr daté du 7/10/2013 qui signale que, puisque la loi Evin du 10 janvier 1991 ne dit rien du vapotage, cela «reste à l'appréciation des employeurs». 
gaz à effet de serre. Et, dans le champ des sciences sociales, la " théorie du risque » désigne traditionnellement l'analyse probabiliste (scientifique) et la gestion (politique) des risques collectifs, et plus particulièrement des risques industriels ou technologiques qui peuvent avoir des conséquences négatives, perturbatrices ou dommageables, sans limites spatiales et parfois sans limites temporelles, sur de larges populations ${ }^{8}$. L'appréhension des risques collectifs se fait souvent à travers le schéma de la guerre, les risques étant assimilables aux ennemis menaçant de l'extérieur des collectivités ${ }^{9}$. Le risque tabagique ne relève pas des risques industriels et semble échapper à ces analyses. Cependant, depuis quelques années, la prise en compte du « risque » comme objet d'étude se double le plus souvent d'un examen des acteurs et des types de discours (expertises médicales; discours médiatiques; pétitions ; actions de consommateurs...) qui interagissent dans l'espace public face à ces nouvelles menaces et les transforment en problèmes publics, véritables « constructions sociales » (affaire de " la vache folle » ou du sang contaminé). Par suite, les approches du risque considèrent encore le plus souvent des vulnérabilités et les interactions entre les acteurs permettant de résoudre des situations de crise. Mais on peut aussi envisager la notion de risque à partir des croyances et des représentations sociales que les individus construisent, voire de la réflexion de sens commun par laquelle ils tentent de minimiser les dangers auxquels ils s'exposent. On peut proposer une approche individualisée du risque avec la cigarette électronique, notamment comme résultat d'une intériorisation d'arguments de santé publique par les individus et comme étant une réponse pragmatique aux risques liés au tabac. Restera à expliquer pourquoi la logique sociétale qui prévaut pour la gestion du risque tabagique semble épargner la cigarette électronique. Les approches du risque disponibles permettent de le situer, en tant qu'objet d'étude, dans le domaine social, mais semblent oublier que l'individu élabore également des stratégies pour répondre à certains risques ${ }^{10}$.

8 Ulrich BECK, Risk Society : towards a New Modernity, Londres, Sage, 1992.

9 C. GILBERT, Le pouvoir en situation extrême, Paris, L'Harmattan, 1992.

10 Dans son ouvrage Société du risque : quelles réponses du politique? (L'Harmattan, Paris, 2007) Sébastien Brunet distingue quatre principales approches du risque : le risque est « une projection dans le futur de la survenance d'un événement dommageable » (p. 19); les études psychométriques (issues de la psychologie cognitive) «dont l'objectif est d'identifier et de mesurer l'influence relative de différentes variables cognitives qui interviennent dans les rapports que les individus entretiennent avec les risques "; une troisième approche d'un point de vue économique ; une dernière mise en perspective du concept de risque renvoie aux théories socioculturelles (parmi lesquelles : la théorie culturelle ; la théorie de la gouvernabilité ; la théorie de la société du risque). Pour la théorie culturelle, analysée par Mary DOUGLAS, « les risques sont considérés comme étroitement déterminés par des modèles culturels de croyances qui rassemblent les différentes perceptions et représentations que les individus ont du monde » (p. 23). Cet auteur développe ainsi une « approche constructiviste-réaliste des risques qui reconnaît que les risques représentent une réalité objective d'un point de vue ontologique. Réalité qui est cependant filtrée à travers différents processus sociaux et culturels, parmi lesquels le mode de connaissance scientifique (...) Elle considère les risques comme une interprétation et une réponse socialement construite par rapport à un danger réel qui existe objectivement, même si la connaissance que l'on en a est nécessairement socioculturellement déterminée. » (p. 29). 
C'est dans la perspective d'une réponse pragmatique aux risques, plutôt que de leur évaluation, que nous souhaitons examiner la possibilité d'une nouvelle approche individualisée du risque, confrontée au terrain et au risque tabagique. Par ailleurs, la problématique des risques se développe aujourd'hui sous deux influences qui se renforcent mutuellement : "d'une part, l'appréhension scientifique et technique du danger et de l'incertitude donne une visibilité sociale de plus en plus grande à tous les risques de la vie humaine ; d'autre part, le corps social envisage de plus en plus systématiquement ses rapports aux autres, aux institutions, à l'environnement en termes de risques (et d'opportunités) ${ }^{11}$. » La notion de risque ne concerne plus uniquement les études des experts et la possibilité de le mesurer objectivement autour de connaissances scientifiques (approche probabiliste et statistique), mais fait désormais partie de la réflexion du sens commun, suite à une intériorisation de l'information. La notion de risque conduit également à penser conjointement un niveau individuel et un niveau collectif : " alors même que les individus cherchent souvent à asseoir leur autonomie face aux institutions, les situations de crise leur rappellent à quel point ils sont insérés dans des ensembles sociaux plus larges, porteurs de contraintes et d'obstacles ${ }^{12}$. » Plus généralement, la notion de risque (et les crises qui lui sont associées) est l'occasion d'interactions sociales et de débats publics, comme le lieu d'une contestation ou d'une inventivité de la part de la société civile. «Il est possible, voire même nécessaire, d'envisager les risques aussi comme des opportunités ; soit comme des événements obligeant les groupes d'acteurs à inventer de nouvelles solutions, à repenser leurs modes "d'être ensemble"13. . L'appréhension des risques par la société civile renvoie ainsi, en réponse aux expertises scientifiques, à des pratiques permettant de les réguler, mais aussi à des stratégies individuelles d'élaboration d'une protection ou à des mécanismes de construction sociale d'un sentiment de sécurité.

Il apparaît que les acteurs sociaux auxquels s'adressent les discours de santé publique font preuve d'autonomie et que l'on peut mettre en évidence des « dimensions symboliques et relationnelles des conceptions et pratiques sociales face au risque ${ }^{14}$. » Claudine Burton-Jeangros souligne qu'une nouvelle manière d'appréhender la santé est apparue au $\mathrm{xx}^{\mathrm{e}}$ siècle, suggérant que « la maladie et l'accident sont des événements évitables, autrement dit qu'ils sont du ressort de l'action humaine, à travers la réduction - au niveau individuel des facteurs de risques identifiés ${ }^{15}$. » Peut-on parler d'une normalisation des esprits face aux problématiques de santé ? Il est, dans tous les cas, permis,

11 Claudine BURTON-JEANGROS, Christian Grosse et Valérie November, Face au risque, introduction, Genève, Georg, 2007, p. 9.

12 Idem, p. 11.

13 Ibidem.

14 Claudine BURTON-JEANGROS, op. cit., p. 184.

15 Ibidem. 
tout au moins, de parler d'une « individualisation des risques ${ }^{16}$, liée à une augmentation de l'information médicale diffusée dans la population ${ }^{17}$. La diffusion des connaissances épidémiologiques affecte aussi les rapports à la santé et à la maladie de diverses manières : "d'une part, ce processus de privatisation des risques suscite une volonté de colonisation du futur, soit le souci de normaliser et maîtriser les événements à venir. D'autre part, les actions de prévention contribuent à "populariser une représentation probabiliste de la maladie" ${ }^{18}$. La distinction entre maladie et santé devenant de plus en plus floue, il y aurait émergence d'un nouvel état intermédiaire, celui d'être « à risque ». Cette incertitude permanente semble effectivement avoir trouvé un certain écho auprès du public qui expérimenterait quotidiennement cette nécessité de se prémunir contre le danger, «l'insécurité dans tous les domaines de la vi $\mathrm{e}^{19}$.» L'auteur poursuit en affirmant que «le discours de santé publique s'inscrit dans un mouvement plus large d'individualisation au sein des sociétés modernes. Il s'appuie en effet sur l'image d'un acteur social autonome - voire d'un consommateur - qui peut effectuer des choix, dont celui de sa santé2 ${ }^{20}$. Cette responsabilisation des individus a-t-elle un fondement éthique ? Certes, l'idée d'un État-providence (qui correspond pour l'auteur à « une forme de socialisation des risques ») « suppose des acteurs évitant les risques au niveau individuel, afin d'assurer la pérennité du système d'assurances sociales. » Et, par ailleurs, « l'idée de prévention, faisant clairement appel à la responsabilité individuelle, est aujourd'hui largement prévalente sur celle de répression ${ }^{21}$. "Toujours selon Claudine Burton-Jeangros, «le discours médical et épidémiologique établit une certaine représentation du risque, constituant celui-ci comme un fait naturel et objectif dont la connaissance doit aboutir à une gestion préventive et rationnelle du danger au niveau individue ${ }^{22}$. " Cependant, certaines critiques peuvent être adressées aux campagnes de santé publique (notamment celles qui ont eu pour objet la prévention du VIH) : certains chercheurs en sciences sociales ont montré que «le risque ne peut pas être strictement pensé en termes individuels, mais qu'il doit au contraire être envisagé dans le cadre d'interactions entre acteurs et au sein des normes et valeurs sociales imprégnant les pratiques sexuelles (...) En examinant les savoirs et pratiques profanes comme des constructions symboliques,

16 Idem, p. 187.

17 Il convient de souligner que cette adresse à l'individu a débuté au XIX siècle avec l'hygiénisme et Claudine Burton-Jeangros précise que « des mesures fondées sur l'idée d'une responsabilité individuelle face à la santé existaient déjà : la lutte contre l'alcoolisme, la prévention de la diffusion des maladies vénériennes, l'éducation des mères quant aux soins à apporter aux nouveaux-nés étaient en effet déjà centrées sur les comportements individuels » (Claudine BURTON-JEANGROS, op. cit., p. 185).

18 Luc BERLIVET, « Une biopolitique » in Jean-Pierre Dozon et Didier Fassin, Critique de la santé publique. Une approche anthropologique, Paris, Balland, 2001, p. 95.

19 Claudine BURTON-JEANGROS, op. cit., p. 188.

20 Ibidem.

21 Ibidem.

22 Idem, p. 189. 
diverses études se sont intéressées aux facteurs sociaux et culturels qui rendent la prévention rationnelle telle qu'elle est pensée par les professionnels inopérante du point de vue des acteurs » (...) Il existe ainsi, selon Claudine Burton-Jeangros « des stratégies (explicites ou implicites) par lesquelles les individus parviennent à faire face aux risques de maladie et d'accident. Il s'agit de s'intéresser à la fois aux interprétations (éléments cognitifs) et aux décisions pratiques (comportements de santé), car ces deux dimensions sont en continuelle interaction et s'influencent réciproquement. » Les risques sont alors envisageables comme des « constructions sociales qui s'élaborent au contact de divers discours (scientifique, médias, entourage) et expériences. La théorie des représentations sociales ou la notion de savoirs profanes s'intéresse au savoir de sens commun et à l'autonomie de celui-ci face au discours médical et épidémiologique. La notion de réflexivité, telle qu'elle est évoquée par Beck et Giddens, renvoie, elle aussi, au statut relatif des énoncés scientifiques : les acteurs, conscients des limites de la science et des incertitudes qu'elle génère, au lieu de les accepter sans discussion, révisent constamment leurs conceptions et pratiques en fonction de nouveaux événements ou connaissances ${ }^{23}$. " En particulier, il revient à Herzlich d'avoir articulé les dimensions individuelle et collective des conceptions sociales : il définit la représentation sociale de la santé et de la maladie comme "l'élaboration psychologique complexe où s'intègrent, en une image signifiante, l'expérience de chacun, les valeurs et les informations circulant dans la société ${ }^{24}$."

\section{Le care, idéologie du mieux vivre ensemble}

La prise en compte du souci de l'autre est au centre de la théorie du care, telle qu'elle a été développée, entre autres, par Joan Tronto ou par Carol Gilligan. Adoptant une posture féministe, Joan Tronto prend appui sur les analyses développées par les philosophes écossais (Hutcheson, Hume et Adam Smith) : elle met ainsi l'accent sur " une morale formulée en termes de sensibilité aux conditions particulières, de sympathie et de bienveillance à l'égard d'autrui ${ }^{25}$.» L'éthique du care peut ainsi être décrite comme une attention ou un soin envers autrui et ceux qui nous entourent. Liane Mozère la désigne encore comme une " proto-morale » qui demande que chacun s'astreigne à " une éducation à la vertu, (au) sens des fins de la vie humaine (et) un sens moral ». Ces qualités, " proches de la conception de la vertu prônée par Aristote, supposent une proximité non seulement physique mais morale aux "autruis" dont on partage le monde et se traduit par une forme de bienveillance de réciprocité qui dépend de la situation dans laquelle se trouvent les individus »: Hume parle de « sympathie étendue » et Adam Smith d'un « sentiment de convenance »,

23 Idem, p. 190.

24 Cité par Claudine BURTON-JEANGROS, op. cit., p. 191.

25 Liane MOZĖRE, « le souci de soi » chez Foucault et le souci dans une éthique politique du care, Le Portique [en ligne], 13-14, 2004, mis en ligne le 15 juin 2007. 
de « décence » ou de « sympathie morale » qui contraignent l'individu à tenir compte de la situation des " autruis ». Dans cet univers, hommes et femmes sont également dotés des mêmes vertus morales et s'obligent, d'une certaine manière, « à adopter activement la place de l'autre ${ }^{26}$. " Tronto définit encore la morale du care comme une «morale contextuelle». Et Gilligan va jusqu'à suggérer une approche gestaltiste en morale. Pour Joan Tronto, il est préférable de se référer à cette autre définition du care : "Une activité caractéristique de l'espèce humaine qui inclut tout ce que nous faisons en vue de maintenir, de continuer ou de réparer notre "monde" de telle sorte que nous puissions y vivre aussi bien que possible. Ce monde inclut nos corps, nos individualités (selves) et notre environnement, que nous cherchons à tisser ensemble dans un maillage complexe qui soutient la vie ${ }^{27}$. » On peut émettre l'hypothèse que nombre des fumeurs qui adoptent la cigarette électronique, songent non seulement à leur santé, mais se soucient aussi d'autrui dans une problématique $\mathrm{du}$ vivre ensemble. Dire que les vapoteurs adoptent la posture du care revient ainsi, en définitive, à dire qu'ils ont intégré le souci d'autrui.

Il semble également que la cigarette électronique répond souvent à une préoccupation écologiste. On peut ainsi lire dans Petit manifeste pour une écologie existentielle de Thierry Paquot qu' " adopter une écologie-attitude revient à s'opposer à la société de consommation, non pas de façon répressive mais ludique, en suggérant des alternatives ${ }^{28}$. » Et l'e-cigarette apparaît bien comme un alternative à l'industrie du tabac et à ses multinationales comme au monopole des buralistes. Plus précisément, cet auteur cite Jean Chesneau, qui décrit les " écogestes " comme des " pratiques civiques de proximité ${ }^{29}$, ce qui s'accorde avec la théorie du care. Félix Guattari a également publié Les Trois Écologies, où « il préconise qu'on adopte un nouveau regard, articulant l'éthique à la politique, sur les trois écologies (environnementale, sociale et individuelle ou subjective), qu'il désigne par l'appellation d'“écosophie" ${ }^{30}$. " Et c'est dans un sens élargi du souci écologique (qui déborde largement la seule prise en compte de l'environnement) qu'il faut aborder l'écologie, en prenant en compte différents niveaux d'action, c'est-à-dire en considérant également les comportements sociaux et les interactions humaines. Ainsi, Thierry Paquot souligne que « depuis la politique énergétique d'un État à l'écogeste de l'écolier, depuis le respect strict des accords internationaux en matière de protection de la nature au choix individuel de pratiquer le tri sélectif des ordures ménagères, il ne faut rien exclure, tout a une importance à des échelles différentes ${ }^{31}$. Et l'auteur de poursuivre : «le fumeur, en jetant

26 Idem.

27 Joan TRONTO, "Care démocratique et démocraties du care », in Pascale Molinier et ali., Qu'est-ce que le care? souci des autres, sensibilité, responsabilité, Paris, Payot, 2009, p. 37.

28 Thierry PAQUOT, Petit manifeste pour une écologie existentielle, Paris, Bourin éditeur, 2007, p. 12.

29 Idem, p. 17.

30 Idem, p. 39

31 Idem, p. 41 
son paquet de cigarettes vide sur le trottoir, se venge du tarif élevé du tabac et des vexations que la société lui impose en le considérant comme un paria à qui l'on réserve des aires bien délimitées pour l'exercice de son "vice" ${ }^{2}$. » La cigarette électronique apporte ici une réponse écologique, puisqu'elle implique la disparition des paquets de tabac comme des mégots, déchets polluants, que ce soit dans la nature comme en ville.

Plus généralement, l'e-cigarette peut être associée à un style de vie, lié à la postmodernité, où l'individu conserve certains plaisirs liés à l'acte de fumer tout en adoptant une démarche " éco-citoyenne " et en construisant une image de soi comme «étant connecté ». Apparue dans les années 2009, elle est associée pour bon nombre de vapoteurs à la généralisation des réseaux sociaux, à la téléphonie mobile liée à internet $(3 \mathrm{G})$, à un style de vie qui repose sur des comportements virtuels et font appel à des objets et des marchandises dématérialisés ${ }^{33}$.

La notion de risque à laquelle la cigarette électronique répond, ne concerne pas uniquement un risque pour la santé, mais aussi un risque pour la qualité de la sociabilité. En utilisant la cigarette électronique, on se conforme à une exigence sociale tout en préservant le rituel et la gestuelle qui font que, comme déjà énoncé, vapoter c'est toujours encore un peu fumer... L'e-cigarette, pharmicone de la pharmacopée censée guérir la société des ravages du tabac est peut-être en passe de devenir un objet culte post moderne. Véritable émoticone du selfcare et du care, elle est peut-être en passe de représenter en termes de sociabilité, ce qu'a été, au tournant des années quatre-vingt, pour la pandémie du sida le petit ruban rouge entrelacé porté sur les boutonnières militantes. Elle est directement liée à la culture généralisée du risque qui s'est développée à la fin du siècle précédent. Sa pratique s’inscrit dans le déploiement de stratégies individuelles nouvelles de respect de soi et d'autrui. Une telle dynamique peut passer inaperçue, mais laisse présager, avec d'autres pratiques du même type, à résonances écologistes et citoyennes, d'importants changements qualitatifs de société en matière d'éthique individuelle et collective. C'est la raison pour laquelle les Sciences de l'Information et de la Communication devraient s'intéresser, avec les moyens de l'enquête et de l'analyse, à cette émergence contemporaine, très significative des transformations de nos sociétés.

\section{BIBLIOGRAPHIE}

AÏACH Pierre, DELANOË Daniel, L'ère de la médicalisation, Paris, Anthropos, 1998.

BECK Ulrich, La société du risque. Sur la voie d'une autre modernité. Paris, Flammarion, 2001.

BRUNET Sébastien, Société du risque : quelles réponses politiques? Paris, L'Harmattan, 2007. 


\section{C\&O n ${ }^{\circ} 45$}

BURTON-JEANGROS Claudine, GROSSE Christian et NOVEMBER Valérie, Face au risque (ouvrage collectif), Genève, Georg, 2007.

DOZON Jean-Pierre et FASSIN Didier, Critique de la santé publique. Une approche anthropologique, Paris, Balland, 2001

ETTER Jean-François, La Vérité sur la cigarette électronique, Paris, Fayard, 2013.

KAZAZIAN Thierry, Il y aura l'âge des choses légères : design et développement durable, Victoire éditions, 2003.

LESOURNE Odile, La genèse des addictions, essai psychanalytique sur le tabac, l'alcool et les autres drogues, Paris, PUF, 2007.

MOLINIER Pascale, Laugier Sandra, Paperman Patricia, Qu'est-ce que le care? Souci des autres, sensibilité, responsabilité. Paris, Payot, 2009.

MOZĖRE Liane, " le souci de soi " chez Foucault et le souci dans une éthique politique du care, Le Portique [en ligne], 13-14, 2004, mis en ligne le 15 juin 2007.

PAQUOT Thierry, Petit manifeste pour une écologie existentielle, Paris, Bourin éditeur, 2007.

POUCHARD Alexandre, Le Monde.fr daté du 7/10/2013.

Résumé : La gestion du risque, en tant que territoire scientifique et conceptuel, s'est principalement développée au début du $\mathrm{XX}^{\mathrm{e}}$ siècle, d'un point de vue statistique, notamment avec la posture assurantielle. Succédant à la prise en charge des populations par le pouvoir souverain et central, cette période historique débouche, avec l'économie du sida, sur un renversement notable. De nos jours, l'orthopraxie étatique est accompagnée par les mouvements du self care et du care. Ces nouvelles pratiques et modes de pensée, liées à l'idée de citoyenneté, annoncent de grands changements sociaux. L'adoption de l'e-cigarette rappelle l'attitude militante de la « safe society » liée à la première pandémie du sida.

Mots-clés : cigarette électronique, risque, nouvelle citoyenneté, orthopraxie étatique, théorie du care, santé publique, éthique.

Abstract : Risk managment, as scientific and conceptual territory, has been developed mainly at the begining of the XXth century, on statistical fashion, notably with the insurance point of view. Succeding to the sovereign and central power welfare of population, this historical period leads with Aid's economy to a singular reversal. State orthopraxis nowadays is alongside selfcare and care. These new practicies and ways of thinking, linked to the idea of citizenship, announce great social changes. The e-cigarette adoption brings to mind a militant attitude of the safe society linked to the first pandemia of Aid.

Keywords : E-cigarette, Risk managment, Ethics, State orthopraxis, Selfcare, care, citizenship, social changes. 